\title{
mtCLIC/CLIC4, a Chloride Channel Protein, Participates in Apoptosis and is Localized to the Inner Membrane of Mitochondria
}

\author{
V.V. Speransky, ${ }^{*}$ K.S. Suh, ${ }^{* *}$ E. Fernández-Salas, ${ }^{* *}$ S.H. Yuspa, ${ }^{* *}$ and A.C. Steven* \\ * Laboratory of Structural Biology, NIAMS/NIH, 50 South Dr. MSC 8025, Bethesda, MD 20892 \\ ** Laboratory of Cellular Carcinogenesis and Tumor Promotion, NCI/NIH, 37 Convent Dr., MSC \\ 4255, Bethesda, MD 20892
}

mtCLIC/CLIC4 (mtCLIC) is a p53 and TNF- $\alpha$ regulated cytoplasmic and mitochondrial protein that belongs to the CLIC family of intracellular chloride channels [1]. Overexpression of mtCLIC reduces mitochondrial membrane potential, releases cytochrome $\mathrm{c}$ into the cytoplasm, and induces apoptosis [2]. We have sought to characterize mtCLIC-induced apoptosis at electron microscopy level, looking for possible ultrastructural changes in mitochondria. We have also used immunoelectron microscopy to localize the mtCLIC protein within the mitochondrion.

S1 primary murine keratinocytes were fixed and thin-sectioned 48 hours following transfection with mtCLIC-GFP. Various stages of apoptosis were observed. Condensation of chromatin into characteristic "crescentic" masses adjacent to the nuclear envelope appeared to be the earliest event (Fig. 1), followed by shrinking of cytoplasm, convolution and blebbing of the plasma and nuclear membranes, and fragmentation of the nucleus. In addition to these known apoptosis markers [3], we noted almost complete loss of tonofilaments. In comparison, non-transfected neighboring cells appeared to have the same amount of tonofilaments as in the control (Fig. 2). Phagocytes being absent in vitro, these preparations also contained abundant post-apoptotic cells in a more advanced state of degeneration, with completely degraded nuclei, ruptured membranes and deteriorating organelles. In apoptotic cells, however, the mitochondria appeared largely unchanged.

To localize mtCLIC, we prepared cryosections of HACAT cells and labeled them with an affinitypurified antibody against C-terminus domain of this protein. Positive labeling of mitochondria was obtained whereas control sections incubated with an unrelated antibody [4] showed complete absence of labeling (data not shown). Consistent with light microscopy and cell fractionation data [1], some sparse label was observed outside mitochondria. This label appeared to be cytosolic and not associated with any membrane-bound compartment. Almost all of the gold particles labeling mitochondria were in close association with cristae or peripheral inner membrane. A few gold particles appeared to be in the matrix without a membrane nearby; however, in such cases, the antigen could well be in a membrane that was cut at an oblique angle and is therefore not visible in the section. There was no significant labeling of the mitochondrial outer membrane. This apparent membrane association, together with putative pore forming and ion transport activity of mtCLIC [2], supports the proposed role for the opening of channels in the apoptotic response [5].

References

[1] E. Fernández-Salas et al., J. Biol. Chem. 247 (1999) 36488.

[2] E. Fernández-Salas et al., submitted for publication.

[3] R.T. Allen et al., J. Pharmacol. Toxicol. Methods 37 (1997) 215.

[4] V.V. Speransky et al., J. Cell Biol. 153 (2001) 1327.

[5] We thank Drs D. Winkler and N. Cheng for providing EM facilities. 

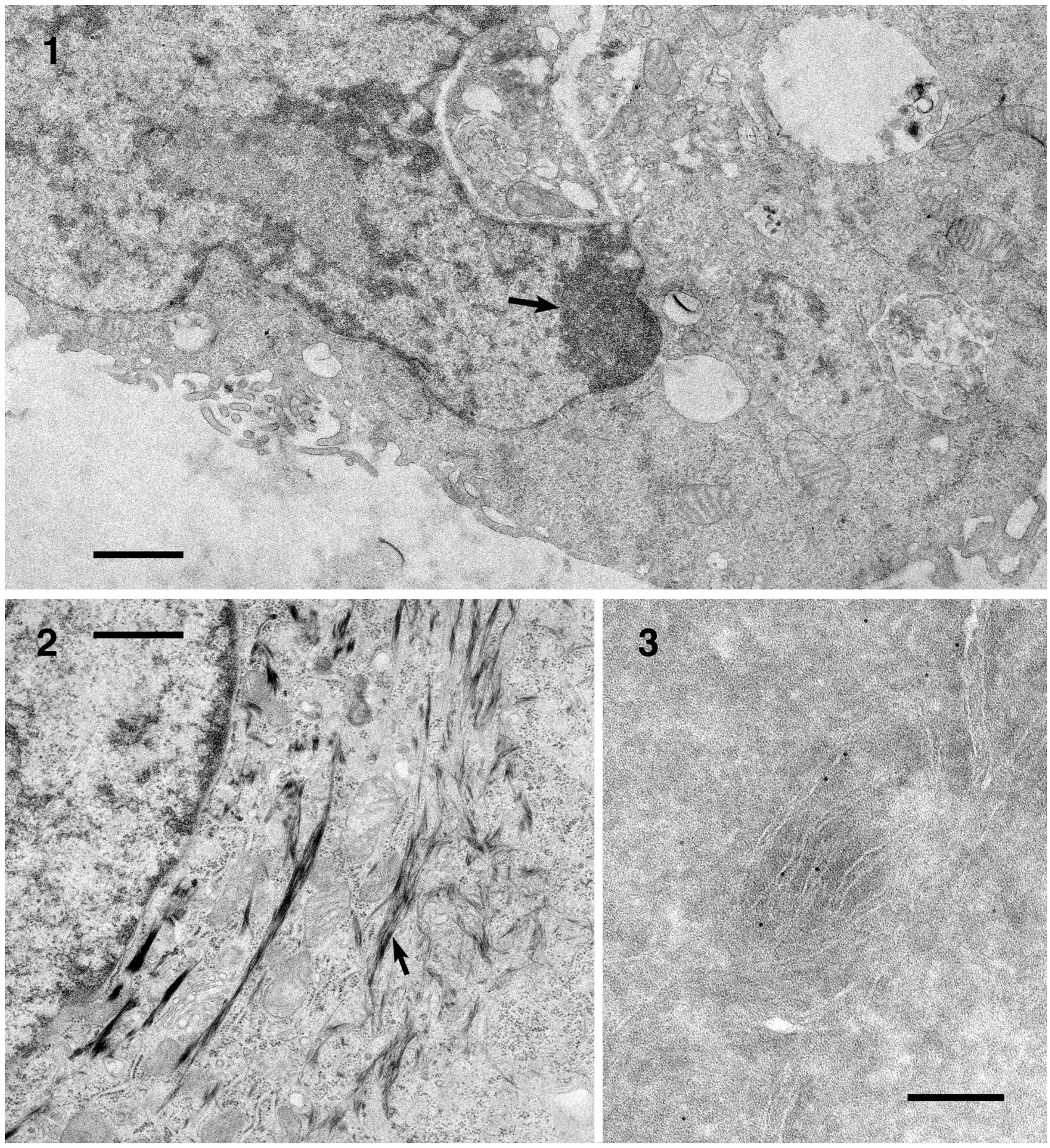

Fig. 1. Early apoptosis in a S1 keratinocyte transfected with GFP-mtCLIC. Chromatin condenses into characteristic marginal "crescentic" masses (arrow). Note absence of tonofilaments, in comparison with the cell in Fig.2. Bar $=1 \mu \mathrm{m}$.

Fig. 2 Control cell transfected with GFP only. Arrow indicates tonofilaments. Bar $=1 \mu \mathrm{m}$.

Fig. 3. Cryosections of HACAT keratinocytes labeled with an affinity-purified antibody against the Cterminal domain of mtCLIC, visualized by Protein A-gold. Gold particles inside mitochondria are in close association with the inner membrane and cristae. $\operatorname{Bar}=0.2 \mu \mathrm{m}$. 